\title{
Trombocitose essencial: o que é essencial saber
}

\section{Essential thrombocytosis: what is vital to know}

Rosane I. Bittencourt ${ }^{1}$

Karin Poncelet ${ }^{2}$

Antonio Carlos C. Almeida ${ }^{2}$

Katia Fassina ${ }^{2}$

Tor G. Onsten ${ }^{2}$
A trombocitose essencial (TE) faz parte do grupo de sindromes mieloproliferativas (SMP) cromossomo Philadelphia(Ph) negativas. Caracteriza-se pela hiperproliferação megacariocítica com consequente trombocitose periférica, favorecendo fenômenos trombo-hemorrágicos. Esta entidade estava esquecida até meados de 2005, quando as recentes publicações sobre as alterações moleculares na atividade da enzima tirosina quinase, JAK2, desencadeou um novo interesse sobre a patogenia, aspectos clínicos e terapêuticos da TE. A identificação das mutações de JAK2 e do gene MPL W515K, W515L e S505N impulsionou a nova proposta da Organização Mundial de Saúde $(O M S)$ para reformular os critérios diagnósticos, reduzindo o número de plaquetas para $450 \times 10^{9} / \mathrm{L}$. O alicerce do tratamento são agentes redutores das contagens plaquetárias: hidroxiureia, anagrelide ou interferon associados à prevenção das complicações trombo-hemorrágicas. Não há um tratamento curativo para a TE, mas despontam perspectivas de que terapias alvo, bloqueadoras da mutação JAK2, possam incrementar o desfecho da doença. Inibidores de JAK2, especificos e inespecificos, estão sendo estudados em fase I e II e parecem promissores num futuro próximo. Rev. Bras. Hematol. Hemoter. 2010;32(2):162-170.

Palavras-chave: Transtornos mieloproliferativos; trombocitose; mutação; contagem de plaquetas.

\section{Introdução}

Em 1951, William Dameshek introduziu o termo síndromes mieloproliferativas (SMP) para designar um grupo de entidades hiperproliferativas com semelhanças clínicolaboratoriais. Agrupou quatro síndromes clássicas: leucemia mieloide crônica (LMC), policitemia vera (PV), trombocitose essencial (TE) e mielofibrose primária (PMF). Nos anos 60, a descoberta do cromossomo Philadelphia $(\mathrm{Ph}+)$ tornou a $\mathrm{LMC}$ uma entidade independente. ${ }^{1}$ As três demais foram relegadas ao grupo das desordens mieloproliferativas cromossomo $\mathrm{Ph}$ negativas, permanecendo num cenário pouco explorado até meados dos anos 2000 .
Entre 1975 e 1986, a TE era confirmada pelo número de plaquetas superior a $1.000 \times 10^{9} / \mathrm{L}$, após a exclusão de trombocitose secundária a causas benignas ou malignas, tipo policitemia vera, leucemia mieloide crônica ou mielofibrose primária. ${ }^{2}$ Em 1986, o Grupo de Estudos de Policitemia Vera (PVSG) revisou os critérios diagnósticos para TE, reduzindo as contagens plaquetárias periféricas para $600 \times 10^{9} / \mathrm{L}$, de forma sustentada além de seis meses, com tendência a fenômenos trombóticos ou hemorrágicos, associadas à franca hiperplasia medular de megacariócitos hiperlobulados e agrupados na ausência de mielodisplasia ou fibrose. Mesmo classificada como doença mieloproliferativa clonal, a TE tem um curso relativamente benigno,

\footnotetext{
${ }_{2}^{1}$ Médica hematologista.

${ }^{2}$ Hematologista. Hospital Luterano - Ulbra - Porto Alegre-RS. Ulbra - Porto Alegre-RS.

Correspondência: Rosane Bittencourt

Hospital das Clinicas de Porto Alegre - Serviço de Hematologia - $2^{\circ}$ andar

Rua Ramiro Barcelos, 2350 - Largo Eduardo Z Faraco

90035-903 - Porto Alegre-RS - Brasil

Tel.:/Fax.: (55 51) 3359-8317

e-mail:rbittencourt@hcpa.ufrgs.br

Doi: 10.1590/S1516-84842010005000021
}

Serviço de Hematologia e TMO do Hospital das Clínicas de Porto Alegre-RS; Serviço de Hematologia do Hospital Luterano - Complexo 
quando as possíveis complicações são controladas, e a sobrevida pode exceder a 15 anos. ${ }^{3,4,5}$

Até 2005, pouco era conhecido sobre a patogênese molecular da TE. Destacava-se a superprodução dos megacariócitos maduros, e sua etiologia estava atrelada basicamente a defeitos no gene do receptor da trombopoetina (c-mpl). A partir das publicações de Joana Baxter, alterações moleculares precisas foram conhecidas e associadas a um clone anormal. ${ }^{6}$ Duas importantes mutações foram descritas: 1) do gene da tirosina quinase citoplasmática, a JAK2 V617F, mutação somática adquirida, presente em média em 50\% dos casos de TE, e 2) do gene receptor da trombopoetina (MPL) (W515L/W515K), que promovem uma vantagem proliferativa celular e estão presentes nos casos de TE JAK2V617F negativa. ${ }^{7}$ Nos dois últimos anos, novas mutações adquiridas estão implicadas na fisiopatogenia das mieloproliferações, porém são fatos recentes, e seus mecanismos não estão completamente elucidados, não deixando claro se causam impacto no desfecho da SMP.

Alguns portadores de TE são assintomáticos, enquanto outros experimentam sintomatologia vasomotora inespecífica, como eritromelalgia, cefaleia, distúrbios visuais ou parestesias de extremidades. Os sintomas são em maior monta repercussões trombóticas em órgão alvos. Angina, infarto do miocárdio, acidente vascular cerebral (AVC) e embolia pulmonar podem representar a primeira manifestação de trombocitose. As manifestações hemorrágicas são menos frequentes e mais associadas ao número de plaquetas superior a $1.000 \times 10^{9} / \mathrm{L}$, variando de epistaxe e gengivorragia até hemorragias digestivas ou sangramentos em sistema nervoso central (SNC), algo mais raro. ${ }^{8}$

Em 2008, uma nova proposta da Organização Mundial de Saúde (OMS) organizou os critérios diagnósticos, definindo parâmetros para estratificação de riscos e posterior tratamento das SMP ${ }^{4,5}$ Uma importante modificação foi relativa ao número plaquetário, reduzindo de $600 \times 10^{9} / \mathrm{L}$ para $450 \times 10^{9} /$ $\mathrm{L}$ plaquetas. O diagnóstico precoce permite medidas profiláticas nos pacientes de maior risco para eventos deletérios. Para alguns autores, os pacientes de menor risco podem ficar sem terapia, já outros defendem benefícios com o uso de aspirina em baixas doses. ${ }^{8}$

O objetivo deste manuscrito é realizar uma breve atualização da TE: aspectos moleculares, clínicos, os critérios OMS 2008 para o diagnóstico e a abordagem na prática diária.

\section{Epidemiologia}

A incidência de TE nos Estados Unidos e Europa varia de 0,6 a 2,5/100.000 pessoas/ano. ${ }^{8}$ A frequência atual é maior do que a registrada nas décadas passadas, fato possivelmente explicado por: a) maior automação de exames; b) maior acesso dos profissionais às informações e c) mudança na estratégia de diagnóstico conforme a OMS, reduzindo o número de plaquetas de $600 \times 10^{9} / \mathrm{L}$ para $450 \times 10^{9} / \mathrm{L} .{ }^{59}$ No Brasil, ainda não há um registro epidemiológico específico para as TE ou para as doenças mieloproliferativas individualizadas.

A idade média ao diagnóstico é em torno dos 60 anos, sendo possível ocorrer dos 18-90 anos. Há casos restritos na infância. ${ }^{10,11} \mathrm{~A}$ trombocitose primária é extremamente rara em crianças, geralmente este diagnóstico só fica estabelecido na segunda década de vida, em média aos 11 anos. ${ }^{12}$ Há discreta prevalência no sexo feminino $(2 \mathrm{~F}: 1 \mathrm{M}){ }^{8}$ A ocorrência de TE durante a gravidez tem sido reportada em várias séries da literatura. ${ }^{13}$

\section{Etiologia e base molecular}

A essência comum nas desordens mieloproliferativas é a superprodução de células maduras descendentes da célula progenitora, unidade formadora de colônias de elementos mieloides, monócitos, megacariócitos, eritrócitos, eosinófilos e basófilos (UFC-GMME). O mecanismo responsável pelos eventos adquiridos ainda não está completamente estabelecido na TE e nem nas demais mieloproliferações $\mathrm{Ph}$ negativas. ${ }^{14}$

Por décadas, atribuiu-se a vantagem proliferativa megacariocítica a fatores relacionados à trombopoetina e ao seu receptor celular (c-Mpl), regulados pelas citocinas: Interleucina 3(IL-3), IL-6 e IL-11, que juntamente com a resistência aos inibidores da megacariopoese interagem com o microambiente medular, produzindo e maturando megacariócitos exageradamente. A hiperplasia megacariocítica acarreta consequente plaquetose, responsável pela redução proteolítica dos multímeros do fator de von Willebrand, promovendo uma síndrome de Von Willebrand adquirida, reforçada pela ativação das plaquetas com defeito no metabolismo do ácido araquidônico e redução dos receptores de prostaglandina D2, mantendo o tromboxane2 em franca atividade, facilitando sangramentos. ${ }^{15}$

Existem defeitos genéticos adquiridos envolvidos na etiologia da TE, mas pouco se sabe sobre sua dinâmica no ciclo celular. Em 35\% a 70\% das TE, ocorre a mutação do gene da janus kinase 2, JAK2V617F, responsável pela fosforilação da molécula transdutora de sinais ativadores de transcrição (STAT), um gatilho do ciclo celular. ${ }^{6}$ Pode-se dizer que a proteína JAK2 serve de intermediária entre os receptores da membrana e as moléculas de sinalização para desencadear a ação do ciclo celular. ${ }^{16}$ As diferenças qualitativas e quantitativas na mutação JAK2V617F explicam a heterogeneidade entre as desordens mieloproliferativas, configurando a possibilidade de que mutações adquiridas em outros genes também são determinantes no comportamento biológico da doença. ${ }^{17}$ A descoberta de três novas mutações somáticas na região justamembrana, no códon 515 do MPL (W515L, W515K, S505N) explica defeitos nas vias de sinalização das moléculas STAT3, STAT5, na proteína ativadora do mitogênio (MAPK) e na fosfatidil-3 kinase ATK (PI3K/AKT), conce- 
Tabela 1. Fenômenos trombóticos

1) Sistema cardiovascular
Gangrena digital
Trombose venosa profunda
Trombose coronária
angina
IAM
Hipertensão
Pericardite
2) Sistema nervoso central e periférico
Ataque isquêmico transitório (AIT)
AVC isquêmico
Hipertensão intracraniana
Cefaleia - tipo enxaqueca
Zumbidos
Disartria
Síncope
Convulsões
Parestesias de extremidades
3) Trato respiratório
Hipertensão pulmonar
4) Trato digestivo
Trombose de veia porta (síndrome de Budd-Chiari)
Trombose de mesentérica
5) Ocular
Oclusão veia retina
Amaurose fugaz
Diplopia
Escotomas
6) Sistema gênito-urinário
Oclusão artéria renal
Insuficiência renal aguda
Priapismo

Tabela 2. Fenômenos hemorrágicos

1) Trato digestivo Hematêmese Melena

2) Pele e mucosas

Petéquias

Equimoses

Epistaxe

Gengivorragia

3) Sistema gênito-urinário Hematúria

dendo um ganho de função e uma vantagem proliferativa aos megacariócitos. ${ }^{18,19}$

O gene supressor Ten-Eleven Translocation 2 (TET)2, encodado no cromossomo 4, pode sofrer deleções ou mutações em 12\% dos portadores de SMP. Um rearranjo no cromossomo 4q24 promove a mutação somática no gene supressor TET2 em progenitores imaturos, sendo um evento primário nas SMP, inclusive precedendo a mutação JAK2 na evolução da doença. ${ }^{20}$ Alguns autores referem uma relação entre as alterações no gene (TET) 2 com quadros de PV e TE em fase de evolução para mielofibrose ou leucemia aguda. ${ }^{19-22}$
Manifestações clínicas

O quadro clínico da TE é bastante variável. Cerca de $25 \%$ a $35 \%$ dos pacientes são assintomáticos e descobrem a trombocitose acidentalmente. Em outros, a sintomatogia é frusta, manifesta apenas pelos sinais vasomotores tipo eritromelalgia ou acrocianose. As Tabelas 1 e 2 discriminam as manifestações trombóticas e hemorrágicas respectivamente de acordo com os órgãos alvo.

\section{Diagnóstico}

A condição inicial para suspeitar de TE é a persistência de um número elevado de plaquetas em sangue periférico. Contagens maiores de $450 \times 10^{9} / \mathrm{L}$ plaquetas merecem uma investigação.

O diagnóstico inclui a deteção de hiperplasia megacariocítica, revelando megacariócitos maduros, hiperlobulados, agrupados na medula óssea, além da exclusão de causas sistêmicas capazes de elevar plaquetas. ${ }^{15}$ Estudos moleculares para a deteção de mutações são uma ferramenta valiosa para o diagnóstico. A pesquisa da mutação JAK2V617F é positiva entre 35\%-70\% dos casos de TE. ${ }^{6} \mathrm{~A}$ JAK2 mutada favorece a leucocitose, predispondo a tromboses. ${ }^{23,24}$ As mutações do gene MPL (W515L, W515K e S505N) incidem em 10\% de TE JAK2V617F negativa, estando associadas ao maior índice de anemia. ${ }^{1}$

Nas tabelas 3 e 4 estão os critérios diagnósticos convencionais e nova proposição da OMS 2008 respectivamente..$^{25,26}$

Na maioria dos centros brasileiros, os métodos moleculares não fazem parte da rotina, e os critérios convencionais são ainda as bases para o diagnóstico de TE.

Tabela 3. Critérios convencionais para diagnóstico de TE antes OMS 2008

1. Contagens de plaquetas superiores a $600 \times 10 \% / \mathrm{L}$ em duas ocasiões diferentes com intervalo mínimo de 30 dias

2. Ausência de causas secundárias de trombocitose

3. Ausência de fibrose medular

4. Ausência de massa eritrocitária elevada

5. Ausência de cromossomo $\mathrm{Ph}+$

6. Presença de esplenomegalia ao exame físico ou pela ecografia do abdome

7. Biópsia de medula óssea hipercelular com hiperplasia megacariócitos

8. Ausência de ferropenia

9. Proteína $\mathrm{C}$ reativa e níveis de $\mathrm{IL}-6$ normais

Todos os critérios de número 1 ao número 5 são imprescindíveis para firmar o diagnóstico de TE. Os demais são importantes e complementam a hipótese diagnóstica.

Fonte: Hoffman R \& Fruchtman S. Essential Thrombocythemia. In: Hoffman Benz Jr E, Shattill S, Furie B, Cohen H, Silberstein L, McGlave P. Hematology. Basic principles and Practice 2005; Elsevier, N .York, 4th edition, Cap 71, p 1277-96 
Tabela 4. Critérios da OMS 2008 para diagnóstico de TE

Contagens de plaquetas superiores a $450 \times 10 \% / \mathrm{L}$

Biópsia de medula óssea com hiperplasia megacariocítica, apresentando grupos de megacariócitos grandes e maduros com núcleos hiperlobulados

Exclusão de outras doenças mieloproliferativas (PV; Mielofibrose e LMC) e de síndrome mielodisplásica

Jak2 positiva ou mutação MPL positiva

Ausência de causas secundárias de trombocitose

Presença de esplenomegalia ao exame físico ou pela ecografia do abdome

Todos os critérios devem estar presentes.

Fonte: Tefferi A, Thiele J, Vardiman J.The 2008 World Health Organization Classification System for Myeloproliferative Neoplasm. Cancer 2009; 3842-47.

Quadro 1. Causas secundárias de trombocitose

Infecções em geral

Pós- esplenectomia

Neoplasias

Doenças inflamatórias

Ferropenia

Outras mileoproliferações

Quadro 2. Diferenças clínico-laboratoriais entre TE e TR

\begin{tabular}{lcc}
\hline Característica & TE & TR \\
\hline Aumento crônico número plaquetas & + & - \\
Doenças sistêmicas associadas & - & + \\
Eventos trombo-hemorrágicos & + & - \\
Esplenomegalia & + & $+/-$ \\
Medula óssea: megacariócitos & + & - \\
aumentados e agrupados & $+/$ & $-+/-$ \\
Alterações citogenéticas & - & + \\
Elevação de proteínas de fase aguda & $+(50 \%$ & - \\
Mutação JAK 2 & $+(10 \%)$ & - \\
Mutação MPL &
\end{tabular}

\section{Diagnóstico diferencial}

No Quadro 1 estão listadas desordens associadas à trombocitose secundária, enquanto no Quadro 2 podem-se observar achados clínicos e laboratoriais úteis na distinção entre TE e trombocitose reacional (TR) secundária a outros processos sistêmicos.

\section{Estratificação de risco}

A TE é uma condição crônica com curso clínico prolongado e de comportamento relativamente benigno, mas que não tem cura. $\mathrm{O}$ manejo clássico está centrado na prevenção das complicações trombóticas e/ou hemorrágicas deletérias. Existem fatores clássicos associados às complicações na TE: a idade, a história pregressa individual ou familiar de fenômenos trombóticos ou hemorrágicos espontâneos e o número plaquetário. Embora a literatura convencional refira risco com plaquetas $>1.000 \times 10^{9} / \mathrm{L}$, após a reclassificação da OMS, há evidências de que plaquetas superiores a $450 \times 10^{9} / \mathrm{L}$ já são suficientes para causar sintomatologia. A tendência atual é considerar as contagens entre $450 \times 10^{9} / \mathrm{L}$ e $600 \times 10^{9} / \mathrm{L}$ plaquetas como determinantes do risco. ${ }^{4,27}$ Regev e colaboradores reportam um estudo onde há associação de manifestações trombóticas e hemorrágicas em 22\% de TE com contagens plaquetárias inferiores a $600 \times 10^{9} /$ L. $^{28}$ Cortelazzo e colaboradores enfatizaram a idade como fator de risco para tromboses num grupo de 100 pacientes com TE. Detectaram tromboses em 1,7\% pacientes/ ano naqueles com menos de 40 anos; $6,3 \%$ pacientes/ano entre $40-60$ anos e $15,1 \%$ pacientes/ano, após 60 anos. ${ }^{29}$ Outra discussão é a inclusão da mutação JAK2V617F como um fator de risco elevado para fenômenos trombóticos, considerando que esta favorece a leucocitose e tromboses nas SMP. ${ }^{30,31}$

O risco de hemorragias é relativamente inferior ao risco de tromboses perfazendo $0,33 \%$ pacientes/ano, predominando fenômenos hemorrágicos de maior porte em pacientes com plaquetas superiores a $1500 \times 10^{9} / \mathrm{L} .^{32}$

A Figura abaixo ilustra a estratificação de riscos baseada nas tendências atuais.

\section{Abordagem}

$\mathrm{Na}$ TE, a abordagem contempla o tratamento de sintomatologia manifesta, a prevenção de fenômenos trombohemorrágicos e a citorredução plaquetária. As tromboses e hemorragias estão relacionadas diretamente ao número exagerado de plaquetas e ao tempo de exposição à trombocitose. ${ }^{29}$ Outros fatores predisponentes à doença cardiovascular, como hipertensão, diabetes, tabagismo, dislipidemia e obesidade, devem ser controlados. O fumo é fator de risco independente para tromboses arteriais. ${ }^{33} \mathrm{~A}$ investigação de trombofilias é pertinente, havendo maior prevalência de anticorpos antifosfolipídeos em TE. ${ }^{34,35}$

\section{Baixo risco}

Há a prerrogativa de que portadores de TE assintomáticos e de baixo risco - idade inferior a 40 anos, plaquetas entre 450 e $600 \times 10^{9} / \mathrm{L}$, ausência de história pregressa de fenômenos trombo-hemorrágicos e inexistência de fatores de risco cardiovasculares - poderiam ser apenas acom-

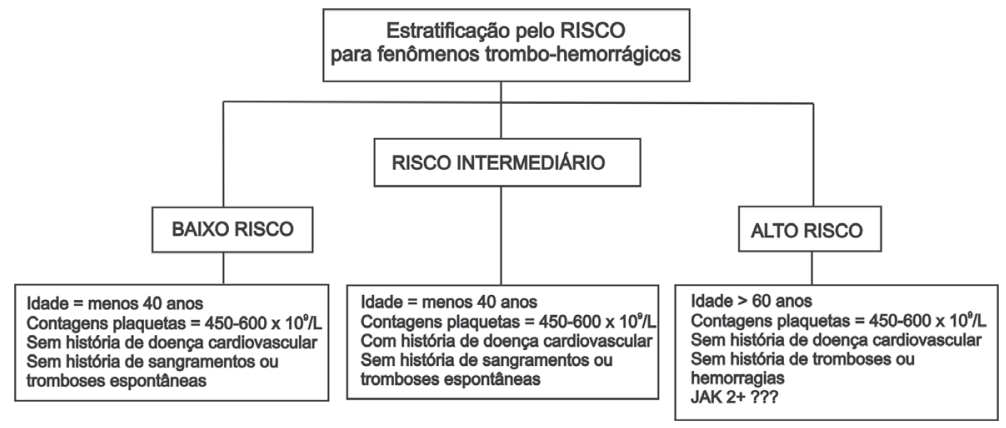


panhados, adotando a conduta watch-and-wait, recomendação grau B de evidência. ${ }^{1,30}$ Entretanto, outros autores defendem que este grupo deve receber aspirina, na dosagem de $100 \mathrm{mg} / \mathrm{dia}$, e manter o acompanhamento clínico e laboratorial a cada seis meses. ${ }^{7,15,36,37}$ Estudos confirmam que aspirina evita as complicações trombóticas e reduz distúrbios microvasculares, tais como a eritromelalgia, acrocianose, cefaleia, tonturas, distúrbios visuais e parestesias em extremidades (palma das mãos e planta dos pés). ${ }^{38,39,40}$

\section{Risco intermediário}

Nestes pacientes, a terapia deve ser individualizada, visando controlar os fatores de risco. Plaquetas superiores a $600 \times 10^{9} / \mathrm{L}$ em presença de fatores cardiovasculares permitem administração de $100 \mathrm{mg} /$ dia de aspirina e/ou agentes redutores da trombocitemia, recomendação grau C. ${ }^{34}$ Os pacientes entre 40-60 anos, expostos a mais de 600 $\mathrm{X} 10^{9} / \mathrm{L}$ plaquetas durante período prolongado de tempo, aumentam o risco de tromboses e merecem terapia. ${ }^{41}$ Não há consenso de qual a melhor recomendação para os pacientes com menos de 60 anos, contagens plaquetárias inferiores a $600 \times 10^{9} / \mathrm{L}$, sem história de fenômenos vasomotores ou trombo-hemorrágicos, mas em vigência de fatores cardiovasculares.

\section{Alto risco}

É uma recomendação grau $\mathrm{A}$, tratar os pacientes acima de 60 anos, com plaquetas além de $600 \times 10^{9} / \mathrm{L}$ e fatores de risco cardiovasculares. A melhor indicação é hidroxiureia e o alvo é reduzir as plaquetas a menos de $450 \times 10^{9} / \mathrm{L} .{ }^{34} \mathrm{~A}$ aspirina não é habitual nos pacientes com plaquetas além de $1000 \times 10^{9} / \mathrm{L}$.

\section{Agentes indicados na trombocitose essencial}

\section{Hidroxiureia}

Há três décadas tem sido o agente de escolha nas SMP, com a finalidade de citorredução. Inibe a síntese de DNA, através de bloqueio da enzima ribonucleoside redutase. A dose inicial preconizada é $15 \mathrm{mg} / \mathrm{kg} / \mathrm{dia}$, podendo alcançar $30 \mathrm{mg} / \mathrm{kg} / \mathrm{dia}$, para reduzir e manter as contagens plaquetárias inferiores a $400 \times 10^{9} / \mathrm{L}$. Leva em média trinta dias para citorreduzir o número de plaquetas, mas pode ultrapassar mais de três meses. ${ }^{34} \mathrm{~A}$ falha na citorredução ocorre em menos de $5 \%$ dos pacientes. ${ }^{7}$ A mielossupressão é o efeito colateral mais preocupante, onde neutropenia e anemia são capazes de interromper a terapia. A monitorização deste efeito, nos dois primeiros meses, exige hemogramas quinzenais. ${ }^{42} \mathrm{Sob}$ aspecto neurológico, pode ocasionar letargia, cefaleia, tonturas, desorientação e, mais raramente, alucinações. Os efeitos gastrintestinais são comuns: estomatite, anorexia, náuseas, vômitos, diarreia e constipação. Na pele ocorrem erupções maculopapulares, hiperpigmentação, eritema facial e periférico, ulcerações ou agravamento de úlceras pré-existentes.
Os níveis séricos de ureia e creatinina devem ser monitorados, com recomendação de ajustar doses conforme a depuração de creatinina: $10-50 \mathrm{~mL} / \mathrm{min}$ administrar $50 \%$ da dose e $<10 \mathrm{~mL} / \mathrm{min}$ somente $20 \%$. Outros efeitos indesejáveis são febre, calafrios, mal-estar e astenia. ${ }^{43}$

A presença da mutação JAK2 pode interferir na resposta à hidroxiureia, conforme demonstrado no estudo MRC PT1, onde os JAK2 positivos foram mais sensíveis, com significativa queda no número de plaquetas. ${ }^{8,43}$

A questão da mutagenicidade induzida pela hidroxiureia ainda gera discussões. A hipótese que induz à transformação em leucemia aguda não foi absolutamente comprovada. ${ }^{45,46}$ $\mathrm{O}$ fato de inibir a enzima difosfato-ribonucleotidase, evitando o reparo de DNA, aumenta a instabilidade genética e abre a possibilidade de carcinogênese, porém não existem estudos em humanos comprovando este fato. Seu uso não é recomendado durante gestação porque atravessa a barreira placentária.

Cerca de $15 \%$ dos pacientes apresentam intolerância, efeitos colaterais não controláveis ou falha de resposta à hidroxiureia, evoluindo para terapias de segunda linha como interferon e anagrelide. A Tabela 5 ilustra os motivos de falha de resposta à hidroxiureia. ${ }^{47}$

Tabela 5. Motivos de falha com hidroxiureia

\begin{tabular}{|c|c|c|c|}
\hline $\mathrm{Hb}$ & Plaquetas & Leucócitos & Hidroxiureia \\
\hline \multirow{4}{*}{$\begin{array}{l}\mathrm{Hb}<10 \mathrm{~g} \\
\text { Anemia }\end{array}$} & $>450.000$ & & qualquer dose $\mathrm{HU}$ \\
\hline & $>450.000$ & $\begin{array}{l}<2.500 \\
\text { Leucopenia }\end{array}$ & qualquer dose $\mathrm{HU}$ \\
\hline & $\begin{array}{l}>600.000 \\
\text { Trombocitose } \\
\text { Persistente }\end{array}$ & & $\begin{array}{l}\text { Após } 3 \mathrm{~m} \text { com HU } \\
\text { mais de } 2 \mathrm{~g} / \mathrm{dia} \text { ou } \\
2,5 \mathrm{~g} / \mathrm{Kg} / \mathrm{dia}\end{array}$ \\
\hline & & & $\begin{array}{l}\text { Efeitos colaterais } \\
\text { intoleráveis: úlceras } \\
\text { em pele e/ou } \\
\text { mucosas; febre }\end{array}$ \\
\hline
\end{tabular}

Fonte: Barosi G, Besses C, Bigegard G, Briere J, Cervantes F, Finazzi G, Gisslinger $\mathrm{H}$ et al. A unified definition of clinical resistance/intolerance to hydroxyurea in essential thrombocythemia: results of a consensus process by an international working group. Leukemia 2007;21: 277-80

\section{Interferon}

É um agente imunomodulador com propriedades biológicas capazes de interferir na diferenciação celular. Pelo seu efeito antiproliferativo no megacariócito, tem sido empregado para reduzir o número de plaquetas, porém esta constitui uma indicação fora da bula (off label). Nunca foi licenciado para uso em TE, nem pelo FDA e nem pela Anvisa no Brasil. ${ }^{48}$

É um agente de difícil administração e sua recomendação é bastante limitada. Além da aplicação por via subcutânea, carrega importantes paraefeitos: síndrome flu-like 
(febre, calafrios, mialgia, rash cutâneo, cefaleia) náuseas, vômitos, diarreia, fadiga, depressão, aumento de triglicerídios, efeitos autoimunes, como artrites, hipotireoidismo ou tireoidite. Mais ou menos 50\% dos pacientes interrompem a terapia por conta dos efeitos colaterais incontroláveis. As doses empregadas são varáveis: desde 6 até 70 milhões semanais, porém a dose inicial recomendada é $3 \mathrm{MU} /$ dia até alcançar resposta. Devem ser realizados hemogramas semanais no primeiro mês desta terapia. ${ }^{47}$ Nenhum efeito leucemogênico tem sido reportado com o IFN ${ }^{49}$ É contraindicado nos portadores de doenças de tireoide ou em pacientes psiquiátricos. Seu uso é permitido durante a gestação, pois não atravessa a barreira placentária. ${ }^{50}$

\section{Anagrelide}

É um composto imidazo-quinazolina, com ação seletiva para a linhagem megacariocítica. Age sob o megacariócito na fase pós-mitótica do seu desenvolvimento, inibindo sua maturação. Não tem ação no DNA, não sendo mutagênico. ${ }^{51}$ A dose inicial preconizada é $0,5 \mathrm{mg}$ a $1 \mathrm{mg} / \mathrm{dia}$ (um ou dois comprimidos) na primeira semana, com ajustes semanais de $0,5 \mathrm{mg}$ até atingir resposta hematológica satisfatória, podendo levar entre 4 a 12 semanas. A dose máxima não deve exceder a $10 \mathrm{mg} /$ dia. Foi considerado efetivo para a redução de trombocitemia, reduzindo indiretamente as complicações trombo-hemorrágicas. Cacciola e colaboradores descrevem sua experiência com o anagrelide, enfocando a redução dos níveis plaquetários e a reversão em $20 \%$ da mutação JAK2 nos pacientes positivos ao diagnóstico. ${ }^{52}$ Ressalta-se que este é um estudo pequeno e que os resultados necessitam confirmação.

No Serviço de Hematologia do Hospital Luterano - Porto Alegre (RS) tem-se uma pequena experiência com o anagrelide em TE. Em um grupo de 12 pacientes, predominando o sexo feminino (9F:3M), idade mediana de 58 anos(32-70 a), com plaquetas superiores a $600 \times 10^{9} / \mathrm{L}\left(612-1500 \times 10^{9} / \mathrm{L}\right)$ após falha ao tratamento com hidroxiureia, foi prescrito anagrelide como segunda opção terapêutica. Houve 75\% (9/12pacientes) de resposta, com dose média diária de $3 \mathrm{mg} /$ dia (seis comprimidos). Na maioria, as plaquetas reduziram a $450 \times 10^{9} / \mathrm{L}$ na média de 4 meses. Os principais efeitos indesejáveis foram diarreia, palpitações, taquicardia e cefaleia, resolvidos com redução de doses. Dois pacientes não toleraram a medicação e interromperam o tratamento. Um paciente necessitou associação de anagrelide + hidroxiureia para controlar a trombocitose.

$\mathrm{O}$ anagrelide possui um efeito inotrópico positivo, aumentando a frequência cardíaca e a pressão arterial, podendo desencadear palpitações, crise hipertensiva, ou retenção hídrica. Seus efeitos colaterais mais frequentes são relacionados ao trato gastrointestinal: intolerância gástrica, náuseas, vômitos, diarreia. Importante cefaleia, anemia leve, vertigens e tonturas são associadas ao anagrelide. ${ }^{31} \mathrm{~A}$ maioria dos efeitos acontece dentro do primeiro mês da terapia, reduzindo a ocorrência à medida que o tratamento avança..$^{53}$ A incidência dos paraefeitos parece maior em indivíduos com mais de 60 anos. ${ }^{54}$

Recomenda-se avaliação cardiológica através de eletrocardiograma previamente e durante o tratamento. Pacientes com história de cardiopatia isquêmica ou arritmias devem ser avaliados criteriosamente antes da introdução desta terapia. $\mathrm{O}$ anagrelide atravessa a barreira placentária, podendo ter potencial teratogênico, estando contraindicado na gestação. ${ }^{29}$

\section{Outras terapias}

$\mathrm{Na}$ impossibilidade da administração das três drogas mais estudadas em TE - hidroxiureia, interferon e anagrelide - restam possibilidades, como os agentes alquilantes melfalano e o bussulfano, porém, sendo terapias de exceção, existem poucos estudos referindo alguma efetividade com esta aplicação. ${ }^{8}$ As modalidades de transplante de célulastronco hematopoéticas(TCTH) não são consideradas terapias convencionais em TE, entretanto o TCTH alogênico pode ser relevante nos casos de evolução de TE para mielofibrose ou leucemia aguda. ${ }^{55}$

\section{Trombocitose na gestação}

Pacientes com TE são predominantemente do sexo feminino, favorecendo algumas vezes o diagnóstico durante a gestação. A literatura reporta em torno de 300 casos de TE e gestação. ${ }^{56} \mathrm{Em} \mathrm{TE}$, a gravidez não é recomendada, mas também não é contraindicada, embora exija maior atenção às possibilidades de complicações, havendo alerta de que os riscos para mãe e feto são aumentados. ${ }^{57}$ Cerca de $50 \%$ das gestações resultam em parto normal a termo, mas há maior incidência de abortos espontâneos no primeiro trimestre. As publicações revisadas reportam uma taxa de abortos espontâneos entre $25 \%$ a $50 \%$, além de maiores índices de morte intrauterina, retardo do crescimento, pré-eclampsia e parto prematuro..$^{57,58}$

A concomitância de trombofilia e TE na gestação eleva os riscos para eventos trombo-embólicos. Há sugestão de que a presença da mutação JAK2617V/F possa contribuir para eventos vasculares na gestação. ${ }^{58}$

Em condições normais, a própria gravidez induz ao estado protrombótico decorrente de alterações hormonais fisiológicas no sistema da coagulação. Adicionalmente, na $\mathrm{TE}$, aumentam riscos de processos trombóticos podendo afetar a mãe e o feto. ${ }^{59} \mathrm{~A}$ literatura ressalta que, durante o segundo trimestre, acontece um declínio espontâneo das contagens plaquetárias. ${ }^{8,32}$

Também nas gestantes a estratificação de risco deve ser aplicada. Gestantes consideradas de risco intermediário devem ser avaliadas individualmente para definição da melhor conduta. É recomendação grau $\mathrm{C}$ que a presença de tromboses, hemorragias ou abortos de repetição induza ao uso 
de aspirina em baixa dosagem. Se for o caso, a aspirina deve ser interrompida sete dias antes do parto. Gestantes de alto risco com contagens de plaquetas além de $600 \times 10^{9} / \mathrm{L}$, história de tromboses ou complicações hemorrágicas, complicações em gestações anteriores ou fatores positivos para trombofilia (Fator V Leiden/anticoagulante lúpico/deficiência das proteínas $\mathrm{C}, \mathrm{S}$ ou antitrombina III) possuem a indicação de receber heparina de baixo peso molecular durante o terceiro trimestre, e se houver necessidade de reduzir as contagens plaquetárias, o interferon é a única droga permitida, recomendação grau B. ${ }^{32}$

\section{Perspectivas atuais e futuras}

O que é essencial hoje na TE é reconhecer a sintomatologia e os parâmetros diagnósticos de acordo com os novos critérios estabelecidos pela OMS em 2008, a fim de precocemente identificar os riscos e introduzir as medidas mitigadoras. $\mathrm{O}$ aprendizado sobre as desordens mieloproliferativas $\mathrm{Ph}$ negativas avançou bastante, o que permitiu a ampliação na abordagem, no entanto ainda não existem indícios de cura, permanecendo este campo em franco desenvolvimento.

A descoberta das mutações dos genes JAK2 e MPL alavancou as pesquisas para encontrar bloqueadores moleculares, mas não podemos ignorar que estas alterações reconhecidas não são os únicos eventos moleculares responsáveis pela TE, e mesmo que os agentes anti-JAK2 contribuam para a melhora do quadro, possivelmente ainda não proverão a cura.

A Tabela 6 mostra os agentes em questão e as fases dos estudos clínicos. ${ }^{60}$

Até que novas tecnologias possam ser incorporadas, o foco na TE mantém-se na redução das contagens do número de plaquetas e na prevenção das complicações trombohemorrágicas. A estratificação pelo risco deve nortear a decisão terapêutica. Agentes citorredutores (hidroxiureia, anagrelide, interferon) associados à aspirina em baixa dosagem e a correção de fatores de risco cardiovasculares ainda são as medidas concretas capazes de estabilizar o quadro clínico.

Tabela 6. Agentes anti-Jak2 em estudos clínicos

\begin{tabular}{ccccc}
\hline & Agente & Fase & via & Alvo primário \\
\hline Seletivos & INCBO18424 & $\|$ & Oral & JAK2 \\
& XL019 & $\|$ & Oral & JAK2 \\
Não & TG101348 & $\|$ & Oral & JAK2 \\
seletivos & MK00457 & $\|$ & Venosa & Aurora kinase \\
& AT9283 & I/I & Venosa & Aurora kinase \\
& VX-680 & I & Venosa & Aurora kinase \\
& CEP 701 & II & Oral & FLT3 \\
& Vorinostat & $\|$ & Oral & Inibidor HDAC \\
\hline
\end{tabular}

\begin{abstract}
Essential thrombocythemia (ET) is an acquired myeloproliferative Philadelphia negative disorder characterized by megakaryocytic hyperproliferation and persistent peripheral thrombocytosis with a tendency of thrombosis and hemorrhages. This entity was forgotten until 2005, when the recent identification of somatic mutations such as JAK2V617F and MPL W515L/K triggered off interest in the molecular pathogenesis, clinical aspects and therapeutic approach of ET. The presence of molecular mutations changed the diagnostic criteria proposed by the World Health Organization, and nowadays the platelet count for which ET should be considered has dropped to $450 \times 109$ /L. Treatment is given according to risk stratification: in cases with high risk platelet reduction, therapy using drugs such as hydroxyurea, interferon or anagrelide is chosen. There is no drug known to cure ET and the current therapy is either to prevent thrombohemorrhagic events or reductions in the platelet count. The identification of the JAK2V617F mutation has opened an opportunity to develop new therapeutic target. JAK2 inhibitors are promising for the treatment of ET in the near future. Rev. Bras. Hematol. Hemoter. 2010; 32(2): 162-170.
\end{abstract}

Key words: Myeloproliferative disorders; thrombocytosis; mutation; platelet count.

\section{Referências Bibliográficas}

1. Vannucchi AM, Guglielmelli P, Tefferi A. Advances in undestanding and management of myeloproliferative neoplasms. CA Cancer J Clin. 2009;59(3):171-91.

2. Laszlo J. Myeloproliferative disorders (MPD): myelofibrosis, myeloscrerosis, extramedullary hematopoiesis, undifferentiated MPD, and hemorrhagic thrombocythemia. Semin Hematol. 1975; 12(4):409-32.

3. Murphy S, Iland H, Rosenthal DS, Laszlo J. Essential thrombocythemia: an interim report from the Polycythemia Vera Study Group. Semin Hematol. 1986;23(3):177-82.

4. Tefferi A, Thiele J, Orazi A, Kvasnicka H, Barbui T, Hanson C, et al. Proposals and rationale for revision of the World Health Organization diagnostic criteria for polycythemia vera, essential thrombocythemia, and primary myelofibrosis: recommendations from an ad hoc international expert panel. Blood. 2007;15(4): 1092-7.

5. Tefferi A, Thiele J, Vardiman JW. The 2008 World Health Organization classification system for myeloproliferative neoplasms: order out of chaos. Cancer. 2009;115(17):3842-7.

6. Baxter EJ, Scott LM, Campbell PJ, East C, Fourouclas N, Swanton $\mathrm{S}$, et al. Acquired mutation of the tyrosine kinase JAK2 in human myeloproliferative disorders. Lancet. 2005;365(9464):1054-61.

7. Chaligné R, James C, Tonetti C, Besancenot R, Le Couédie JP, Fava $\mathrm{F}$, et al. Evidence for MPL W $515 \mathrm{~L} / \mathrm{K}$ mutations in hematopoietic stem cells in primitive myelofibrosis. Blood. 2007; 110(10):3735-43.

8. Brière JB. Essential thrombocythemia. Orphanet J Rare Dis. 2007 8;2:3. doi:10.1186/1750-1172-2-3.

9. Girodon F, Bonicelli G, Schaeffer C, Mounier M, Carillo S, Lafon I, et al. Significant increase in the apparent incidence of essential thrombocythemia related to new WHO diagnostic criteria: a population-based study. Haematologica. 2009;94(6):865-69. 
10. van Dijken PJ. Essential thrombocythemia in a 5-month-old infant. J Pediatr Hematol Oncol. 1997;19(4):354.

11. Randi ML, Putti MC. Essential thrombocytaemia in children: is a treatment needed? Expert Opin Pharmacother. 2004;5(5): 1009-14.

12. Dame C, Sutor AH. Primary and secondary thrombocytosis in childhood. Br J Haematol. 2005;129(2):165-77.

13. Passamonti F, Randi ML, Rumi E, Pungolino E, Elena C, Pietra D, et al. Increased risk of pregnancy complications in patients with essential thrombocythemia carrying the JAK2 $(617>\mathrm{F})$ mutation. Blood. 2007;110(2):485-89.

14. Murphy S. Diagnostic criteria and prognosis in polycythemia vera and essential thrombocythemia. Semin Hematol. 1999;36 (Suppl 2):9-13

15. Hoffman R, Fruchtman S. Essential thrombocythemia. In: Hoffman R, Benz Jr E, Shattill S, Furie B, Cohen H, SilbersteinL, McGlave P. Hematology Basic Principles and Practice 2005; Elsevier N. York, 4th edition Cap 71 p 1277-96.

16. Goldman JM. A unifying mutation in chronic myeloproliferative disorders. N Engl J Med. 2005;352(17):1744-6.

17. Levine RL, Gilliland DG. Myeloproliferative disorders. Blood 2008;112(6):2190-98.

18. Cazzola M. Molecular basis of thrombocytosis. Haematologica 2008;93(5):646-48.

19. Levine RL, Heaney M. New advances in the pathogenesis and therapy of essential thrombocythemia. Hematology Am Soc Education Program Book. 2008;76-82.

20. Delhommeau F, Dupont S, Della Valle V, James C, Trannoy S, Massé A, et al. Mutation in TET2 in myeloid cancers. 2009; 360(22):2289-301

21. Tefferi A, Pardanani A, Lim KH, Abdel-Wahab O, Lasho TL, Patel $\mathrm{J}$, et al. TET2 mutations and their clinical correlates in polycythemia vera, essential thrombocythemia and myelofibrosis. Leukemia 2009;23(5):905-11.

22. Saint-Martin C, Leroy G, Delhommeau F, Panelatti G, Dupont S, James C, et al. Analysis of the ten-eleven translocation 2 (TET2) gene in familial myeloproliferative neoplasms. Blood. 2009; 114(8):1628-32

23. Kralovics R, Buser AS, Teo SS, Coers J, Tichelli A, Van der Maas AP, et al. Comparison of molecular markers in a cohort of patients with chronic myeloproliferative disorders. Blood. 2003;102(5): 1869-71.

24. Carobbio A, Finazzi G, Guerini V, Spinelli O, Delaini F, Marchioli $\mathrm{R}$ et al. Leukocytosis is a risk factor for thrombosis in essential thrombocythemia: interaction with treatment, standard risk factors, and Jak2 mutation status. Blood. 2007; 109(6):2310-13.

25. Sanchez S, Ewton A. Essential thrombocythemia: a review of diagnostic and pathologic features. Arch Pathol Lab Med. 2006; 130(8):1144-50

26. Harrison CN, Gale R, Machin SJ, Linch DC. A large proportion of patients with a diagnosis of essential thrombocythemia do not have a clonal disorder and may be at lower risk of thrombotic complications. Blood. 1999;93(2):417-24.

27. Lengfelder E, Hochhaus A, Kronawitter U, Höche D, Queisser W, Jahn-Eder M, et al. Should a platelet limit of $600 \times 10(9) / 1$ be used as a diagnostic criterion in essential thrombocythaemia? An analysis of the natural course including early stages. Br J Haematol. 1998; 100(1):15-23.

28. Regev A, Stark P, Blickstein D, Lahav M. Thrombotic complications in essential thrombocythemia with relatively low platelet counts. Am J Hematol 1997;56(3):168-72.
29. Cortelazzo S, Vieiro P, Finazzi G, DEmilio A, Rodeghiero F, Barbui $T$. Incidence and factors for thrombotic complications in a historical cohort of 100 patients with essential thrombocythemia. J Clin Oncol 1990;8(3):556-62.

30. Biergegard G. Long-term management of thombocytosis in essential thrombocythaemia. Ann Hematol 2009;88(1):1-10.

31. Monte-Mór BCR, Costa FF. A mutação JAK 2 V617F e as síndromes mieloproliferativas. Revista Bras Hematol Hemoter. 2008;30(3): 241-8.

32. Barbui T, Barosi G, Grossi A, Gugliotta L, Liberato L, Marchetti M, et al. Practice guidelines for the therapy of essential thrombocythemia. A statemet from the Italian Society of Hematology, the Italian Society of Experimental Hematology and the Italian Group for Bone Marrow Transplantation. Haematologica. 2004; 89(2):215-32

33. Jantunen R, Juvonen E, Ikkala E, Oksanen K, Anttila P, Ruutu T. The predictive value of vascular risk factors and gender for the development of thrombotic complications in essential thrombocythemia. Ann Hematol. 2001;80(2):74-8.

34. Harrison CN, Donohoe S, Carr P, Dave M, Mackie I, Machin SJ. Patients with essential thrombocythaemia have an increased prevalence of antiphospholipid antibodies which may be associated with thrombosis. Thromb Haemost. 2002;87(5):802-07.

35. Sacchi S, Vinci G, Gugliotta L, Rupoli S, Gargantini L, Martinelli V et al. Diagnosis of essential thrombocythemia at platelet counts between 400 and 600x10(9)/L. Haematologica. 2000; 85(5):49295.

36. Tefferi A. Essential thrombocythemia, polycythemia vera, and myelofibrosis: Current management and the prospect of targeted therapy. Am J Hematol. 2008;83(6):491-7.

37. Campbell PJ, Green AR. Management of polycythemia vera and essential thrombocythemia. Hematology Am Soc Hematol Educ Program 2005;201-08.

38. van Genderen PJ, Mulder PG, Waleboer M, van de Moesdijk D, Michiels JJ. Prevention and treatment of thrombotic complications in essential thrombocythaemia: efficacy and safety of aspirin. $\mathrm{Br}$ J Haematol 1997;97(1):179-84.

39. Michiels JJ, Koudstaal PJ, Mulder AH, van Vliet HH.. Transient neurologic and ocular manifestations in primary thrombocythemia. Neurology 1993;43(6):1107-10.

40. van Genderen PJ, Michiels JJ, van Strik R, Lindermans J, van Vliet HH. Platelet consumption in thrombocythemia complicated by erythromelalgia: reversal by aspirin. Thromb Haemost. 1995;73(2): 210-14.

41. Regev A, Stark P, Blickstein D, Lahav M. Thrombotic complications in essential thrombocythemia with relatively low platelet counts. Am J Hematol 1997;56(3):168-72.

42. Stevens MR. Hydroxyurea: an overview. J Biol Regul Homeost Agents. 1999;13:172-75.

43. Cortelazzo S, Finazzi G, Ruggeri M, Vestri O, Galli M, Rodeghiero F, et al. Hydroxyurea for patients with essential thrombocythemia and a high risk of thrombosis. N Engl J Med. 1995 Apr 27;332(17): 1132-6.

44. Campbell P, Scott LM, Buck G, Wheatley K, East CL, Marsden J, et al. United Kingdom Myeloproliferative Disorders Study Group; Medical Research Councill Adult Leukemia Working Party; Australasian Leukemia and Lymphoma Group: Definition of subtypes of essential thrombocythaemia and relation to polycythaemia vera based on Jak2 V617F mutation status: a prospective study. Lancet 2005;366(9501):1945-53.

45. Barbui T. Indications for lowering platelet numbers in essential thrombocythemia. Semin Hematol. 2003;40(Suppl. 1):22-5. 
46. Weinfeld A, Swolin B, Westin J. Acute leukaemia after hydroxyurea therapy in polycythaemia vera and allied disorders: prospective study of efficacy and leukaemogenicity with therapeutic implications. Eur J Haematol. 1994;52(3):134-9.

47. Barosi G, Besses C, Birgegard G, Briere J, Cervantes F, Finazzi G, et al. A unified definition of clinical resistance/intolerance to hydroxyurea in essential thrombocythemia: results of a consensus process by an international working group. Leukemia 2007;21(2):277-80.

48. Wadenvik H, Kutti J, Ridell B, Revesz P, Jacobsson S, Magnusson B, et al. The effect of alpha-interferon on bone marrow megakaryocytes and platelet production rate in essential thrombocythemia. Blood. 1991;77(10):2103-08.

49. Merup M, Aberg W, Löfvenberg E, Svensson E, Engman K, Paul C, et al. Symptoms, symptoms distress and health-related quality of life in patients with polycythaemia vera or essential thrombocythaemia during treatment with interferon-alpha. Acta Oncol. 2002;41(1):50-55.

50. Birgegård G, Björkholm M, Kutti J, Lärfars G, Löfvenberg E, Markevärn B, et al. Adverse effects and benefits of two years of anagrelide treatment for thrombocythemia in chronic myeloproliferative disorders. Haematologica. 2004;89(5):520-27.

51. Petitt RM, Silverstein MN, Petrone ME. Anagrelide for control of thrombocythemia in polycythemia and other myeloproliferative disorders. Semin Hematol. 1997;34(1):51-4.

52. Cacciola E, Di Francesco E, Pezzela F, Tibullo D, Cacciola R. Effect of anagrelide on JAK2 mutational status in patients with essential thrombocythemia. Clin Leukemia 2008;2(4):272-74.

53. Steurer M, Gastl G, Jedrzejczak WW, Pytlik R, Lin W, Schlögl E, et al. Anagrelide for thrombocytosis in myeloproliferative disorders: a prospective study to assess efficacy and adverse event profile. Cancer 2004;101(10):2239-46.

54. Fruchtman SM, Petitt RM, Gilbert HS, Fiddler G, Lyne A; Anagrelide Study Group. Anagrelide: analysis of long-term efficacy, safety and leukemogenic potential in myeloproliferative disorders. Leuk Res. 2005;29(5):481-91.
55. Anderson JE, Sale G, Appelbaum FR, Chauncey TR, Storb R. Allogeneic marrow transplantation for primary myelofibrosis and myelofibrosis secondary to polycythaemia vera or essential thrombocytosis. Br J Haematol. 1997;98(4):1010-16.

56. Beard J, Hillmen P, Anderson CC, Lewis SM, Pearson TC. Primary thrombocythaemia in pregnancy. Br J Haematol. 1991; 77(3):371-74.

57. Elliot Ma, Tefferi A. Thrombocythaemia and pregnancy. Best Pract Res Clin Haematol. 2003;16(2):227-42.

58. Passamonti F, Randi ML, Rumi E, Pungolino E, Elena C, Pietra D, et al. Increased risk of pregnancy complications in patients with essential thrombocythemia carrying the JAK2 $(617 \mathrm{~V}>\mathrm{F})$ mutation. Blood. 2007;110(2):485-89.

59. Bates SM, Ginsberg JS. Trombosis in pregnancy. Curr Opin Hematol 1997:4(5):335-37.

60. Pardanani A. JAK2 inhibitor therapy in myeloproliferative disorders: rationale, preclinical studies and ongoing clinical trials. Leukemia. 2008:22(1):23-30.

Avaliação: Editor e dois revisores externos

Conflito de interesse: sem conflito de interesse

Recebido: 05/10/2009

Aceito após modificações: 19/10/2010 